\title{
Architecture for Safe Human-Robot Collaboration: Multi-Modal Communication in Virtual Reality for Efficient Task Execution
}

\author{
$1^{\text {st }}$ Beibei Shu ${ }^{1}$ \\ beibei.shu@uit.no
${ }^{I}$ Department of Industrial Engineering UiT The Arctic University of Norway Narvik, Norway

\author{
$2^{\text {nd }}$ Gabor Sziebig ${ }^{1,2}$ \\ gabor.sziebig@sintef.no \\ ${ }^{2}$ Department of Production Technology \\ SINTEF Manufacturing \\ Raufoss, Norway
}

\author{
$3^{\text {rd }}$ Roel Pieters ${ }^{3}$ \\ roel.pieters@tuni.fi \\ ${ }^{3}$ Department of Automation and \\ Hydraulic Engineering \\ Tampere University \\ Tampere, Finland
}

\begin{abstract}
Task-sharing and Human-Robot Collaboration has gained increased attention with the widespread commissioning and usage of collaborative robots. However, recent studies show that the fenceless collaborative robots are not as harmless as they look like. In order to study HumanRobot Interaction scenarios, in a safe manner, we propose to execute the scenario in Virtual Reality simulation and afterwards implement it in real robotic applications (supervised from VR). In addition, this simulated world allows ad-hoc modifications and easy prototyping of different multi-modal communication forms. In this paper we propose an architecture for safe human-robot collaboration and describe a use-case: task of nut screwing, which is executed by the human and the robot together. The nut is hold by the human and the screw is screwed into the nut by the robot (as this part is the repetitive part of the task). The task can be executed in the VR simulation with different input and feedback channels (multi-modal) in order to identify the most efficient communication way between the human and the robot. The different input and output channels are presented in detail.
\end{abstract}

Keywords-Human-Robot Collaboration, industrial robot, simulation, virtual reality

\section{INTRODUCTION}

Interaction between robots and humans has been gaining increasing interest in research [1-3]. When considering a collaborative scenario in industrial setting much research aims at a task where human and robot complement each other to increase productivity and safety [4-6]. The main challenges to tackle are therefore to increase acceptability from operators and to provide natural collaboration between human and robot $[7,8]$. For both challenges, assumptions are made on the environment and what information appears in it (multi-modal communication) [9]. A closed world assumption states that the world model contains everything the robot needs to know. This in contrast to the open world problem, where new events and new knowledge may appear.
When relying on such closed world view, it is crucial that sufficient sensing modalities are applied such that the complete world can be measured and modelled. While this assumption does not take into account the communication to other agents beside the robot, a clear connection to knowledge representation and sharing can be made.

Since the need of high efficiency and high productivity, the using of industrial robot is increasing in manufacturing and assembling processes. However, not all processes can be automated and human is still needed in the creative tasks, to adapt unpredictable changes e.g. cable assembly on a production line [10]. In this direction, the collaboration of humans and robots seems a promising solution that allows sharing both workplaces and tasks [11].

The remaining part of the paper is organized the following: in Section 2, the related research in HRC, HRI and VR is presented. While in Section 3 the basic idea is described. In Section 4, the proposed architecture is presented and in Section 5 the task execution in VR is detailed. The paper is summarized in Section 6.

\section{RELATED RESEARCH}

\section{A. Human-Robot Collaboration (HRC)}

The collaborative operation is defined as a state in which purposely designed robots work in direct cooperation with a human within a defined workspace [12]. Based on ISO/TS 10566:2016 the collaborative workspace was defined as the space within the operating space where the robot system (including the workpiece) and human can perform tasks concurrently during production operation [13]. Basically, the idea is that robot cannot hurt a person and the means to protect a person are controlled force and speed, separation monitoring, hand-guiding and safety-rated monitored stop. 
ISO $10218 / 2011$ states that HRC is allowed, if one of the following conditions is satisfied: (a) Velocity of the tool center point (TCP) not exceeding $0.25 \mathrm{~m} / \mathrm{s}$ (b) Maximum dynamic power not exceeding $80 \mathrm{~W}$ (c) Maximum static force not exceeding $150 \mathrm{~N}$ [14]. These conditions may be challenged because they do not take into account the size and shape of the robot, the distance between human and robot and the control strategies $[12,15]$.

\section{B. Human-Robot Interaction (HRI)}

Different HRIs will happen when human collaborate with robot. A new classification strategy has been proposed depending on the level of HRI. According to this approach, most of the possible HRI in industry could be classified into four levels of interaction [16].

\section{Safety in HRC and HRI}

In robot safety, the co-worker trust $[17,18]$ in the robot is important and depends on several factors: system performance, recent errors, co-worker knowledge about the system, and interface usability. High robot speeds, accelerations, and the set minimum distance are also important factors. The robot path automatically adjusted to avoid collision must be predictable by the co-worker to exclude the sense of fear and surprise. While collaborative, fenceless robots are currently being intensively researched, there are very few industrial applications in operation. Recent events have shown how controversial the use of such robots is, and the current state of the discussion between robotics manufacturers and certification authorities [19].

According to ISO 10218-1, a risk analysis of a robotic work cell with human-robot collaboration is necessary to identify relevant risks and the means for safeguarding against them. According to this line of reasoning, there is no "safe robot" as such, as the robot is only one part of a complete system/machine. As an example, even a lightweight robot that moves at slow speeds, has rounded edges, and can stop when collision forces reach a defined level could be used to transport sharp objects. Therefore, a complete analysis of the environment, the defined operation of the robot, as well as of what can result from errors in the control system needs to be carried out for every application.

There are many collaborative robots doing well in safety, such as Baxter [20], Sawyer [21], and the FANUC Robot CR4iA [22]. But in compliance to regulations, these robot's speed is kept low and with low payloads to prevent harmful collisions. However, if the collision risk was minimized then the robot could be operating at a higher speed for a greater part of the production time [23].

As discussed in [24], human operators experiences high mental strain or to put it another way, when the distance between operators and robot is reduced or when the robot moves to operators very fast, the operators will feel not safe.

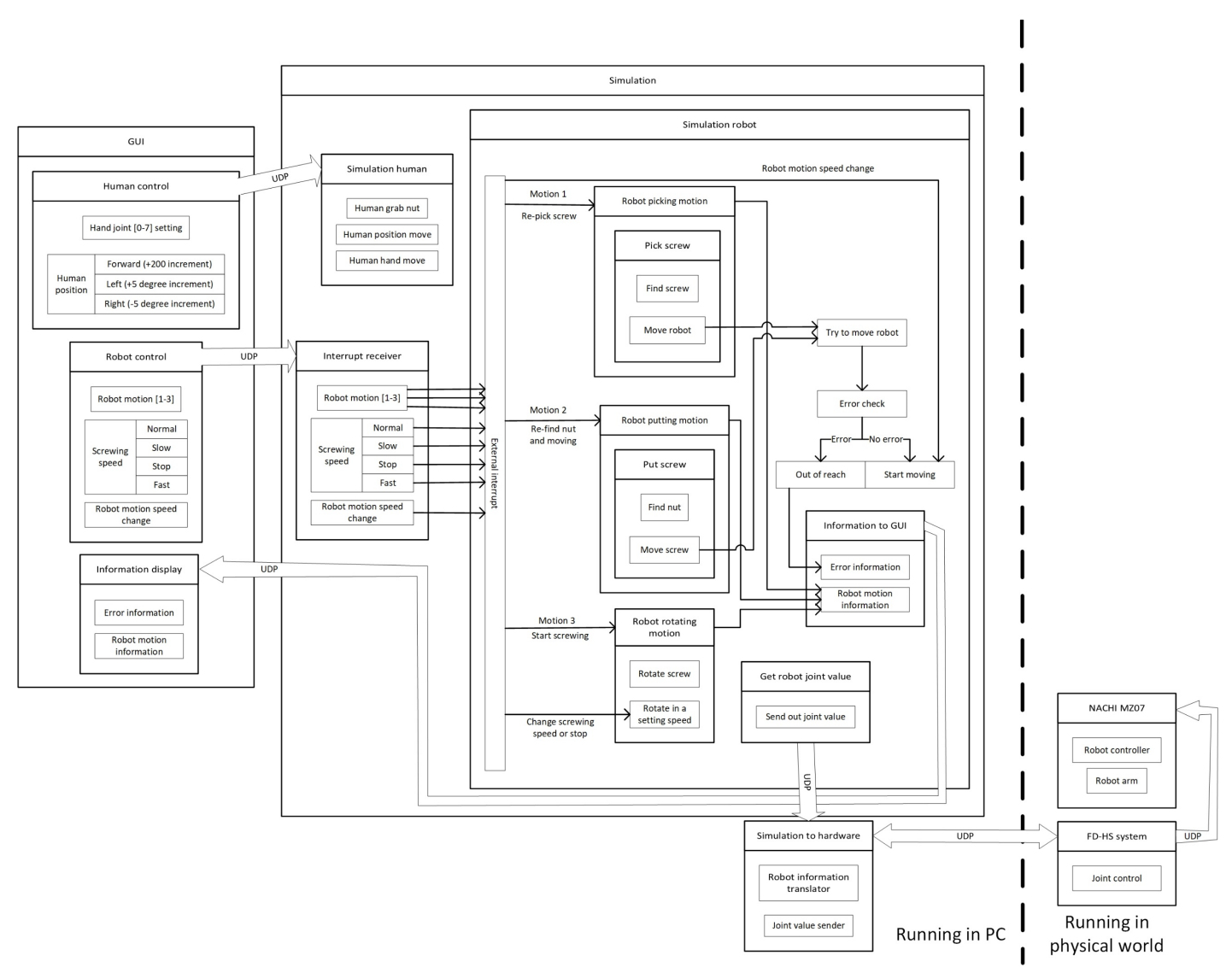

Fig. 1. Experimental collaboration setup 
Vision and distributed computing systems provide low process and evaluation time, allowing for real-time safety assessment and collision avoidance strategies to take place [23]. There are lot of sensors available for HRC systems. Close Human-Robot Collaboration with advanced safety sensors may support speed \& separation monitoring and safety rated monitored stop modes. These might include close proximity sensors, such as pan/tilt/zoom cameras, stereo cameras, depth cameras, projection based-systems [25], and audio/video feedback systems. A better fit for traditional robots (large, high speed, high payload) can be achieved with compliment power \& force limiting functions (PFL robots) [26].

\section{Virtual Reality (VR) simulations}

As the human-robot collaboration is gaining acceptance in the industry, growing demands for change their present robot to collaborative robot [27]. However, changes to the present system are expensive as they frequently require interrupting production to implement and test [4]. Then the test and simulation in advance is needed.

Additionally, a simulation system for hypothesis testing is easy and, another important motivation for pursuing a virtual environment approach against using a real industrial robot is primarily safety of the human, especially when experimenting with different techniques which have not been standardized yet.

Thirdly, accessibility of commercially available robot hardware that may allow such experimentation, e.g. robots with flexible joints and smart controllers, is still restricted; moreover, such hardware is certainly not open enough to accommodate the degree of experimentation that a virtual robot would allow [12].

At last, Virtual Reality collaboration environments offer a solution by enabling high fidelity testing and training of complex manufacturing systems [28].

\section{COLLABORATION IN VIRTUAL REALITY}

Virtual reality offers novel possibilities, when we would like to introduce new type of HRC. While in classical set-up, a robot system must be safe by default, in virtual reality we can allow more complex and not yet safe collaboration methods. One of the tasks, which human solves effectively is placing a nut on a screw. This is however challenging in cases, where the screw and the nut is heavy and a worker needs to do this repetitive task all day long. As this situation requires that the task is shared between the human and the robot, a fully developed automated solution would be needed, on the other hand, with the usage of VR and using it for collaboration solves many of the challenges and simplifies system setup.

However the VR simulation does not reflect all the aspects if a human would be with a direct contact with a real robot. For this purpose, we need to investigate the multi-modal communication possibilities in the specific HRI scenarios. In Fig. 2. this HRI scenario is detailed with all the input and outputs from different aspects.

\section{PROPOSED ARCHITECTURE}

The presented system (as seen in Fig. 1.) is used to simulate robot operation and connect the VR simulation with physical world. Following is the detailed explanation of each block. The setup of the system can be divided into two groups: running on PC and running in physical world (divided by dashed line in the figure).

\section{A. Components running on $P C$}

There are three programs running in PC. The first one is a Graphic User Interface (GUI) in which programmed by Python script's Tkinter module [29]. The GUI is used for controlling the simulated human and robot in the simulation software, and it is communicating with the simulation software by sending/receiving UDP socket. The second one is the industrial simulation software Visual Components [30]. The software not only just give user a real $3 \mathrm{D}$ world to monitor the system's running, but also calculating robot joint's moving and send it out to physical world. The last one is a Python script used for the communication of the simulation and the physical world.

1) Graphic User Interface (GUI): The Graphic User Interface (as seen in Fig. 2.) which can be divided into three parts based from different functions.

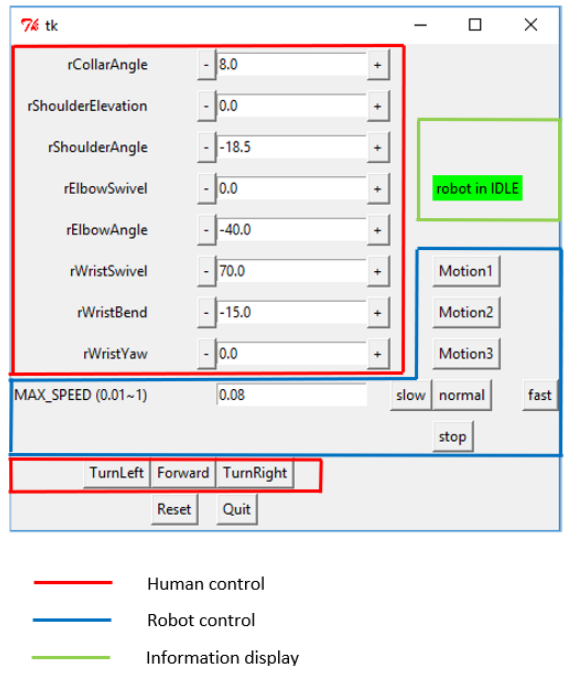

Fig. 2. Graphic User Interface

There are Human control function, Robot control function and Information display function. Explanation in the following:

2) Simulation software: In this sub-section, the detailed explanation of the simulation software will be presented. The simulation software Visual Components (as seen in Fig. 3.) is well designed which in default include a varies of objects in software library, such as the robot NACHI MZ07 and the human worker. 


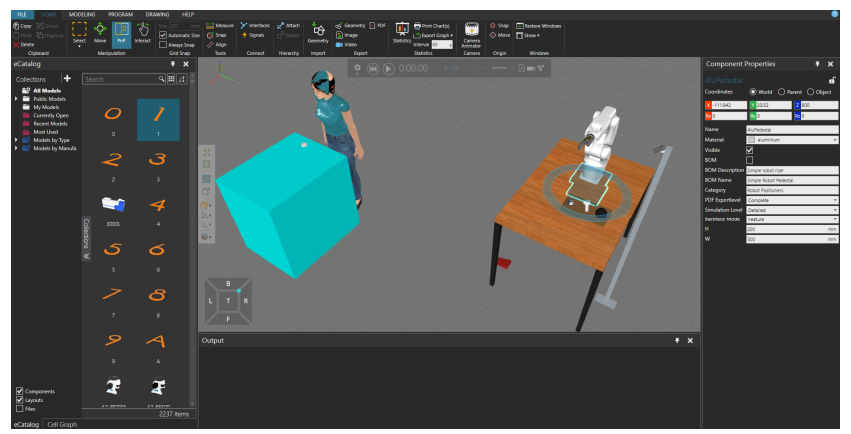

Fig. 3. Simulation in Visual Components

Based on function, we divide the simulation software into 3 parts: simulation human, interrupt receiver and simulation robot.

a) Simulation human: The software offered a Python script application programming interface (API) which can control very detailed motion of the simulation human object (as seen in Fig. 3.). But in this system, only the method to control right hand, the method to control position and the method to grab object are needed. The method to control right hand offered the access to the 7 joints of right arm (as seen in Fig. 1.), the method to control position offered the access to moving human forward and turning right/left, and the last method offered the access to attach object into right hand so the right hand can "grab" object and moving. The simulation human receives UDP socket from GUI then performs the requested motion.

b) Interrupt receiver: The interrupt receiver (as seen in Fig. 3.) is used for breaking robot's present motion and changing it to new motion. The interrupt receiver script must have a carrier except robot itself, it is not specified to any certain object but in this case, we put it in the table. The receiver will receive the robot control signal by UDP socket from GUI and forward it to robot immediately by trigging a robot external interrupt signal. The reason why we design this interrupt receiver is that the robot object is also a program and this program cannot run in multi thread, so the robot itself cannot have a separate thread to listen Ethernet's UDP socket and interrupt robot's present motion then update it into a new motion.

c) Simulation robot: The simulated robot (as seen in Fig. 3.) is combined by a MZ07 robot, a gripper and a pedestal. Inside the simulation software, these components are logically connected so we can regard these components as one single object.

3) Simulation to software: This is a Python script connects simulation and hardware since the FD-HS system cannot direct communication with simulation software. The script will translate the joints value from degree into a special format which FD-HS system understand. And the script also ensures the joint value incrementation will not too big which may damage the robot arm, since the FD-HS system has no interpolator function.

\section{B. Physical world}

There is an option for the system connecting VR simulation with physical world, synchronizing the real robot's movement with simulation robot. Two hardware systems are using in physical world besides PC, there are FD-HS system and NACHI MZ07 robot. The logical and physical connection is shown below (as seen Fig. 4 and Fig. 5.).

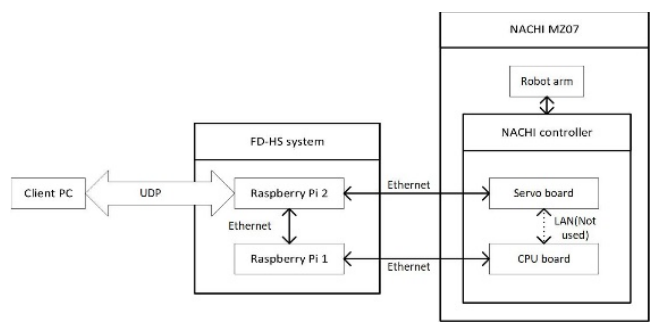

Fig. 4. Logical connection of FD-HS system and NACHI MZ07 robot

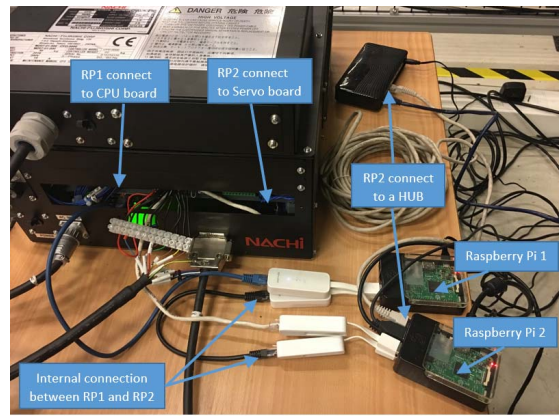

Fig. 5. Physical connection of FD-HS system and NACHI controller

1) FD-HS system: The FD High Speed (FD-HS) interface replaces the original internal direct Ethernet connection of the NACHI controller, thus the communication going from the CPU board through the Raspberry Pi 1 and Raspberry Pi 2 to the Servo board and vice versa, as seen in Fig. 10. Raspberry Pi 2 has the UDP interface to the application clients, while in this system we connect the interface to a HUB. By using FDHS system, we can easily access each joint's value and change it.

2) NACHI MZ07: NACHI MZ07 [31] is a standard 6 joints ultra high-speed industrial robot. Same as other industrial robots, the robot also has a robot arm and a controller. There are 6 servo motors in the arm and the motor driver located in the Servo board of controller. The interpolation, trajectory calculation and user interface are offered by CPU board.

\section{TASK EXECUTION IN VR}

The goal with architecture is to find the most efficient method for human-robot collaboration through virtual reality with using the 4 different input and the 4 feedback methods. The input methods were selected based on the interaction design principles [32] and the feedback methods based on human senses [33]. 


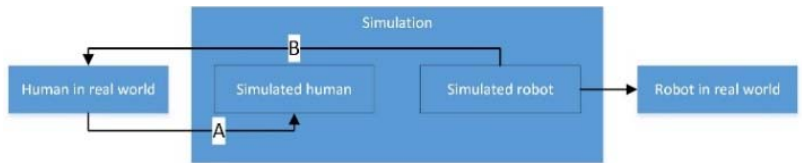

Fig. 6. Overview of Human-Robot Collaboration in experimental setup

Overview of Human-Robot Collaboration in experimental setup is shown in Fig. 6, in which the Simulation block represents the simulation environment including a simulated human and a simulated industrial robot. The movement of the simulated human is based on the input from the operator in the real world (as highlighted with the arrow A), and the feedback to the operator is based on the simulated robot motion and state (as shown with arrow B).

The four different input methods (arrow A) are:

\section{A. Input by $G U I$}

using keyboard and mouse. A well-designed GUI will be presented in front of operator. Operator can move each single joint of simulated human arm by click GUI button.

\section{B. Input by measurement arm}

using a special tool. A measurement arm will be presented in front of operator. Measurement arm constantly output the coordinate of arm tip. Operator can move the nut position in simulation by moving the measurement arm in real world.

\section{Input by 6 DOF mouse}

also using keyboard and mouse but in an advanced way. 6 DOF mouse can output the 3D coordinate directly. Operator can move the simulated nut directly by moving mouse.

\section{Input by motion capture system}

this is using nature interaction. The motion capture system can get all the human operator's arm joint value, and then directly feedback to the simulation, so the simulated human's arm will act as same as real world.

TABLE I. OVERVIEW OF INPUT METHODS

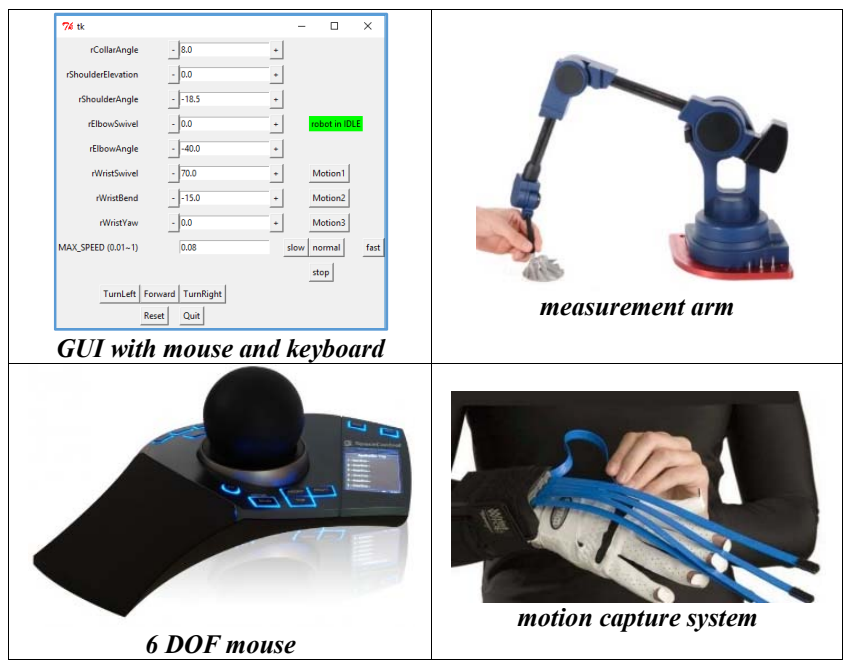

The human senses which can be using in this experiment are only hearing and vision, but the information representation can be different. So, we are using one audio method and three visual methods.

The four different feedback methods (arrow B) are:

\section{A. Audio}

In the simulation, program can make sound to inform operator, so operator will understand which state robot is running in. Fig. 7 shows the shape of audio wave, in which, " $A$ " is in beeping and " $B$ " is in silence. Combination of A and $\mathrm{B}$ form one basic cycle of beeping. By changing the time of "A" and "B" can change the beeping frequency. In the present system, eight zones (zone 0 to zone 7) are defined based on the screw-nut distance, and each zone will trigger one beeping sound with certain frequency, in the meantime the color ring will change into certain color, see in Table II.

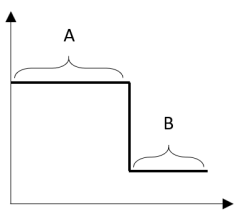

Fig. 7. Shape of beeping audio wave

TABLE II. ZONE, BEEPING AND COLOUR

\begin{tabular}{|l|c|c|c|}
\hline \multirow{2}{*}{$\begin{array}{c}\text { Zone } \\
\text { Number }\end{array}$} & \multicolumn{2}{|c|}{ Beeping Cycle (ms) } & \multirow{2}{*}{ Color } \\
\cline { 2 - 3 } & Beeping & Silence & \\
\hline 0 & 1000 & $\approx 1$ & Red \\
\hline 1 & 200 & 200 & Orange \\
\hline 2 & 300 & 300 & Yellow \\
\hline 3 & 400 & 400 & Green \\
\hline 4 & 400 & 600 & Deep blue \\
\hline 5 & 200 & 1000 & Blue \\
\hline 6 & 200 & 2000 & Pink \\
\hline 7 & 0 & $\infty$ & White \\
\hline
\end{tabular}

\section{B. Visual (text label)}

In the simulation, a label with text message inside will be presented beside the robot. Operator can read the text, acquire the detailed information of robot (as shown in Fig. 8).

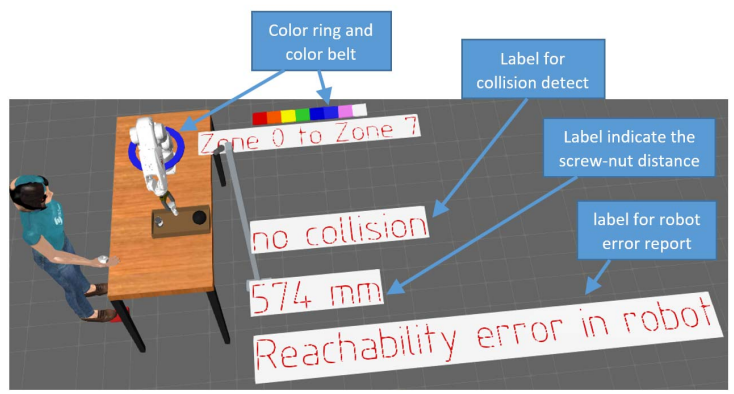

Fig. 8. Label feedback from simulation

There are two labels in the simulation used to feedback the collision state and robot error report respectively. The label for collision detect will only show two states: "no collision" or "collision happen". The label for robot error report can display all the errors listed in Table III. 
TABLE III. ROBOT ERRORS LIST

\begin{tabular}{|l|c|}
\hline State Number & Robot Errors \\
\hline 1 & Reachability error in robot \\
\hline 2 & One or more joints exceeding limits \\
\hline 3 & Singularity detected \\
\hline 4 & Reachability and joint limit errors \\
\hline 5 & Singularity and reachability errors \\
\hline 6 & Singularity and joint limit errors \\
\hline 7 & Singularity, joint limit and reachability errors \\
\hline 8 & Other error \\
\hline 9 & No errors \\
\hline
\end{tabular}

\section{Visual (special sign)}

Several special signs beside the robot will show robot states. The sign can be many shapes (circle, square or triangle) and many colours (as shown on Fig 8, in this example it is circle shape -- "color ring").

\section{Visual (distance)}

The screw-nut centre distance will be updated into a label in a frequency $10 \mathrm{~Hz}$ (as shown on Fig 8 , "label indicate the screw-nut distance").

\section{CONCLUSIONS}

An architecture for safe Human-Robot Collaboration has been presented in this paper. The architecture can be used to supervise tasks, which are hard to control from safety perspective. The presented task is: nut screwing, which is executed by the human and the robot together. The nut is hold by the human and the screw is screwed into the nut by the robot (as this part is the repetitive part of the task). The task can be executed in the VR simulation with different input and feedback channels (multi-modal) in order to identify the most efficient communication way between the human and the robot, which were presented in detail.

\section{REFERENCES}

[1] M. A. Goodrich and A. C. Schultz, "Human-Robot Interaction: A Survey," Foundations and Trends ${ }^{\circledR}$ in Human-Computer Interaction, vol. 1, no. 3, pp. 203-275, 2008.

[2] A. Maria Bauer, D. Wollherr, and M. Buss, Human-Robot Collaboration: a Survey. 2008, pp. 47-66.

[3] G. Michalos, S. Makris, J. Spiliotopoulos, I. Misios, P. Tsarouchi, and G. Chryssolouris, "ROBO-PARTNER: Seamless Human-Robot Cooperation for Intelligent, Flexible and Safe Operations in the Assembly Factories of the Future," Procedia CIRP, vol. 23, pp. 71-76, 2014/01/01/ 2014.

[4] C. Heyer, "Human-robot interaction and future industrial robotics applications," in 2010 IEEE/RSJ International Conference on Intelligent Robots and Systems, 2010, pp. 4749-4754.

[5] J. de Gea Fernández et al., "iMRK: Demonstrator for Intelligent and Intuitive Human-Robot Collaboration in Industrial Manufacturing," KI - Künstliche Intelligenz, vol. 31, no. 2, pp. 203-207, 2017/05/01 2017.

[6] T. R. Savarimuthu et al., "Teaching a Robot the Semantics of Assembly Tasks," IEEE Transactions on Systems, Man, and Cybernetics: Systems, vol. 48, no. 5, pp. 670-692, 2018.

[7] E. Coupeté, V. Weistroffer, O. Hugues, F. Moutarde, S. Manitsaris, and P. Fuchs, "New Challenges for Human-Robot Collaboration in an Industrial Context: Acceptability and Natural Collaboration," in Fifth workshop "towards a Framework for Joint Action", iEEE RO-MAN 2016, New York, United States, 2016.

[8] P. Baranyi, B. Solvang, H. Hashimoto, and P. Korondi, "3D Internet for cognitive info-communication," in 10th International Symposium of Hungarian Researchers on Computational Intelligence and Informatics, CINTI 2009, 2009, pp. 229-243.
[9] B. Solvang, G. Sziebig, and P. Korondi, "Multilevel control of flexible manufacturing systems," in 2008 Conference on Human System Interaction, HSI 2008, 2008, pp. 785-790.

[10] J. O. Oyekan et al., "The effectiveness of virtual environments in developing collaborative strategies between industrial robots and humans," Robotics and Computer-Integrated Manufacturing, vol. 55, pp. 41-54, 2019/02/01/2019.

[11] G. Michalos, S. Makris, P. Tsarouchi, T. Guasch, D. Kontovrakis, and G. Chryssolouris, "Design Considerations for Safe Human-robot Collaborative Workplaces," Procedia CIRP, vol. 37, pp. 248-253, 2015/01/01/2015.

[12] E. Matsas, G.-C. Vosniakos, and D. Batras, "Prototyping proactive and adaptive techniques for human-robot collaboration in manufacturing using virtual reality," Robotics and Computer-Integrated Manufacturing, vol. 50, pp. 168-180, 2018/04/01/2018.

[13] ISO 10218-1:2011, 2011.

[14] ISO 10218-2:2011, 2011.

[15] S. Haddadin, M. Suppa, S. Fuchs, T. Bodenmüller, A. Albu-Schäffer, and G. Hirzinger, "Towards the Robotic Co-Worker," in Robotics Research, Berlin, Heidelberg, 2011, pp. 261-282: Springer Berlin Heidelberg.

[16] M. Bdiwi, M. Pfeifer, and A. Sterzing, "A new strategy for ensuring human safety during various levels of interaction with industrial robots," CIRP Annals, vol. 66, no. 1, pp. 453-456, 2017/01/01/ 2017.

[17] J. D. Lee and K. A. See, "Trust in Automation: Designing for Appropriate Reliance," Human Factors, vol. 46, no. 1, pp. 50-80, 2004/03/01 2004.

[18] J. M. Beer, A. Prakash, T. L. Mitzner, and W. A. Rogers, "Understanding robot acceptance," Georgia Institute of Technology2011.

[19] S. Nördinger, "BGHW warnt (doch nicht) vor Roboter," 2014.

[20] L. e Silva, T. M. Tennakoon, M. Marques, and A. M. Djuric, "Baxter Kinematic Modeling, Validation and Reconfigurable Representation," 2016 .

[21] (2018/Nov/15). Rethink robots, Sawyer. Available: https://www.rethinkrobotics.com/sawyer/

[22] (2018/Nov/15). FANUC Robot, Small Collaborative Robot. Available: https://www.fanucamerica.com/cmsmedia/datasheets/Compact $\% 20 \mathrm{C}$ R\%20line\%20up\%20product\%20information_251.pdf

[23] N. Nikolakis, V. Maratos, and S. Makris, "A cyber physical system (CPS) approach for safe human-robot collaboration in a shared workplace," Robotics and Computer-Integrated Manufacturing, vol. 56, pp. 233-243, 2019/04/01/ 2019.

[24] T. Arai, R. Kato, and M. Fujita, "Assessment of operator stress induced by robot collaboration in assembly," CIRP Annals, vol. 59, no. 1, pp. 5-8, 2010/01/01/ 2010.

[25] C. Vogel, M. Poggendorf, C. Walter, and N. Elkmann, "Towards safe physical human-robot collaboration: A projection-based safety system," in IEEE International Conference on Intelligent Robots and Systems, 2011, pp. 3355-3360.

[26] N. Ando, P. T. Szemes, P. Korondi, and H. Hashimoto, "Friction compensation for 6DOF Cartesian coordinate haptic interface," in IEEE/RSJ International Conference on Intelligent Robots and Systems, 2002, vol. 3, pp. 2893-2898 vol.3.

[27] C. Kardos, Z. Kemény, A. Kovács, B. E. Pataki, and J. Váncza, "Context-dependent multimodal communication in human-robot collaboration," Procedia CIRP, vol. 72, pp. 15-20, 2018/01/01/2018.

[28] P. Galambos et al., "Design, programming and orchestration of heterogeneous manufacturing systems through VR-powered remote collaboration," Robotics and Computer-Integrated Manufacturing, vol. 33, pp. 68-77, 2015/06/01/2015.

[29] (2018/Nov/26). Tkinter - Python interface to Tcl/Tk. Available: https://docs.python.org/2/library/tkinter.html

[30] (2018/Nov/27). Visual Components. Available: https://www.visualcomponents.com

[31] (2018/Nov/28). NACHI MZ07. Available: http://www.nachirobotics.com/product/mz07/

[32] A. Blair-Early and M. Zender, "User Interface Design Principles for Interaction Design," Design Issues, vol. 24, no. 3, pp. 85-107, 2008.

[33] R. Moreno and R. Mayer, "Interactive Multimodal Learning Environments," Educational Psychology Review, vol. 19, no. 3, pp. 309-326, 2007/09/01 2007. 